\title{
Geodesic Active Contours with Shape Priors for Segmentation, Disocclusion, and Illusory Contour Capture
}

\author{
Jacob Householder \\ Fredrick Park \\ Mathematics Department, Whittier College, \\ Whittier, CA 90601 \\ Email: jhouseho@poets.whittier.edu
}

December 10, 2019

\begin{abstract}
Image segmentation is the task of finding salient regions of importance in an image. In this work, we take a curve evolution approach to this problem where we deform an initial curve in the inward normal direction with the objective of finding the boundaries of objects in an image. To achieve this, we propose a variational image segmentation model that incorporates a clique based shape signature with a geodesic active contours energy. The model scheme consists of evolving a parametric representation of an active contour to minimize the penalty that the model induces. This penalty is minimized when the curve is on the boundaries of objects in the image, areas with sudden change in pixel intensity i.e. light to dark. We demonstrate successful capture of illusory contours, segmentation of objects in a cluttered background, and segmentation of occluded objects.
\end{abstract}

\section{Introduction}

Image segmentation is an essential task in computer vision where the goal is to partition an image into pertinent regions of interest. These regions are called segments and usually correspond to specific objects in a given image. The segments can be labeled where groups of pixels represent these objects. Segmentation is an important first step in analyzing the composition of an image. For example, in surveillance video, before a suspect can be identified in a scene, they must be separated from the background. If a license plate of a car needs to be read, the region encompassing it must be determined first. There have been many approaches to this task and one of the most popular is the use of variational methods involving active contours; this technique will be the primary focus of this paper. Applications for segmentation are numerous: medical imaging [29, 42, 19], computer vision [33], stereo reconstruction $[25,26,40]$, illusory contour $[43,21,20]$, point-cloud surface reconstruction 
[27], scene reconstruction from range data [13], and data clustering [41]. In this work, we consider the application of edge based segmentation with shape priors on difficult settings that include illusory contours, clutter and, occlusions.

One of the earliest and most successful variational and partial differential equation (PDE) models for image segmentation is the active contour/snake model proposed by Kass and Witkin [23], in which an energy minimizing spline is evolved under external forces to gravitate towards pronounced image features like edges or lines. A subsequent model independent of curve parameterization, based on [23], is the geodesic active contour model (GAC) proposed by Caselles et al. [6] and Kichenassamy et al. [24].

The GAC model has the following formulation:

$$
\min _{C}\left\{J[C]=\int_{0}^{L(C)} g(|\nabla f(C(s))|) d s\right\}
$$

where $C$ is a curve, $L(C)$ its length, and $d s$ is an element of arc length. The function $g$ is an edge detector function that approaches zero near object boundaries and one near more homogeneous regions of a given image $f$. Thus, the energy in (1) can be interpreted as an edge weighted length measurement that penalizes curves that are long or not on the edge of image features. The energy given by (1) is minimized using the gradient descent algorithm, where its functional gradient is used to determine curve evolution velocity. The key idea is that the functional gradient gives the steepest descent direction for the energy, we can then move the curve in this direction to minimize the energy. Given initial curve $C_{0}$, one step of gradient descent can be viewed as: $C_{1}=C_{0}-\delta \nabla J\left[C_{0}\right]$, with $\delta$ a step size. This yields an evolved curve $C_{1}$. Since $-\nabla J[C]$ points in the direction of steepest descent of $J[C]$, we have $J\left[C_{1}\right] \leq J\left[C_{0}\right]$ assuming appropriate regularity on $J[C]$. This is a way of incrementally evolving a contour to minimize an energy.

This behavior can be seen by considering a few cases. If a curve $C$ is near object boundaries, the penalty assigned by (1) is small and small variations of the curve will not result in a smaller penalty, thus no evolution of the curve takes place under gradient descent minimization. In flat regions without image features, the edge detector will be near 1 , and thus variations of the curve with smaller arc length will better minimize the penalty. The minimization scheme will thus move the curve inwards. The GAC model has been popular and useful for many segmentation tasks. Nonetheless, one of the caveats of the model is that under more difficult segmentation scenarios like those involving noise, clutter, or occlusion, as with many standard segmentation models, the model has a tendency to lose accuracy.

A key sub-problem associated with segmentation is that of utilizing shape priors. Here, shape prior segmentation aims to segment objects under more complicated conditions that can involve noise, clutter, or occlusion (partial or complete). By incorporating global information like shape, segmentations can be more successful in these arduous settings. In building an effective shape prior segmentation model, there are some significant hurdles to overcome. The first is the need for a signature to be invariant under rigid motion while also allowing scalability. Second, the signature should also be able to uniquely identify certain classes of shapes. Lastly, it should have the ability to easily integrate into a segmentation model that still allows for an efficient implementation. We give references to some effective shape signatures $[3,4,15]$ and more can be found in the references therein. 
Following the work of Park [34], we propose to incorporate a two dimensional variant of the clique based signature proposed by Kimmel et al. [3, 4, 15] into the GAC model. In certain settings, the edge based segmentation approach can have advantages over a region based one like that found in the Mumford-Shah Model (MS) [30], which in its 2-phase piecewise constant formulation is known as the Chan and Vese model (CV) [8]. Namely, when image contrast variability between background and object is not as differentiated, or when dealing with objects containing only partial edge information like illusory contours. In the latter, region/contrast based approaches tend to be less accurate since illusory contours rely more on geometric information and psychological/vision based interpolation, see [22]. For example, in Fig. 1, the Staples Center is observed in (a) where it contains non-uniform contrast that may have an average value close to that of the background. This, coupled with extensive clutter, yields a difficult segmentation example by standard methods. In Fig. 1 (b), an example of an illusory contour is given. Here, a white square is observed despite having only partial boundary information, i.e. where the corners are present but edge information is incomplete. Most approaches for capturing illusory contours rely on modeling the illusory contour explicitly. In this work, we only utilize shape information coupled with an active contour to capture them. Also, despite using a feature-based approach, we propose a method that can still capture objects under significant clutter by incorporating an edge detector that uses an evolving Gaussian. Successful numerical results will be presented to illustrate the effectiveness of the proposed model in these various difficult segmentation settings.

The closest related work is that of Park [34] where the author utilizes a polygonal implementation of the 2-phase MS functional and a cliques shape prior signature. The author shows successful numerical results for segmenting occluded objects and objects with nonuniform intensities in the presence of clutter. Despite using the same shape signature, the proposed model differs significantly from that in [34] by using the GAC energy instead of the MS energy. A key benefit in the proposed approach is the ability to segment objects that rely more on features instead of object contrast. In particular, the proposed model is effective at segmenting objects containing average intensity similar to that of the background or objects containing only partial boundary information like illusory contours. Other related work are as follows, a model unifying the GAC/snakes, Rudin-Osher-Fatemi denoising [37], and MS model can be found in [1] while shape prior models involving the MS functional can be found in the work by Cremers et al. [10] and Dŏgam et al. [14]. Other work that use deformable models and shape priors using a level set [32] framework can be found in [2, 7, 36, 39, 28]. Models involving explicit descriptors and moments based descriptors can be found in [18] and [16] respectively. Related work using polygonal and B-spline curve representations in conjunction with region-based active contour models can be found in $[9,11,17,38]$. Related work in shape signatures using Fourier descriptors, generalized cone representations, and Legendre moments can be found in [12], [35], and [16] respectively. Finally, a related work on fully convexifying the MS/CV model can be found in [5].

The rest of the paper is organized as follows, in section 2 we introduce the proposed model, while section 3 explains the minimization of it. Section 4 involves the numerical implementation details of the model, while numerical results can be found in section 5 . Finally, conclusions and future work can be found in section 6 . 


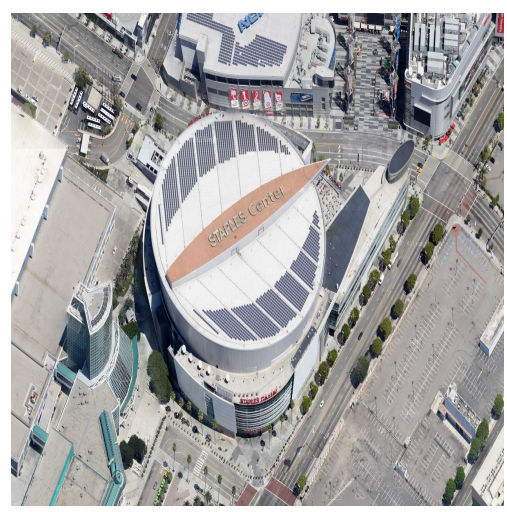

(a) Image with Clutter

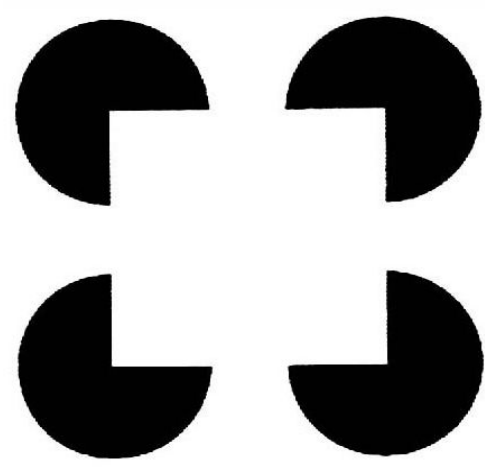

(b) Illusory Contour

Figure 1: Difficult Segmentation Examples. In (a) an image with an object containing nonuniform intensities is present along with clutter. An example of a square illusory contour is seen in (b), where incomplete edge information is present. The intensities of the illusory square match the background.

\section{Proposed Model}

The proposed model builds upon the geodesic active contour model by incorporating shape prior information via a clique. The use of cliques allows the operator of the model to incorporate a scalable, rotation, and translation invariant numerical representation of the desired shape to be segmented (via a library of shape priors). As we will see this allows the model to accurately capture shapes that other models may struggle with such as illusory contours, objects in cluttered backgrounds, and occluded objects.

The model consists of minimizing a function with respect to two terms: a geodesic active contour term and a clique based shape prior term. We will briefly introduce the geodesic active contour model in its continuous form before we define a discrete formulation.

\subsection{Preliminary Notation}

The geodesic active contour model is polygonal based. Therefore finite differences will be used to approximate the derivatives in the discretization and implementation.

Let $\Gamma$ be the collection of polygonal points that represent a curve and $p_{i}$ to be the $i$ th point on this curve as follows: $\Gamma=\left\{p_{i} \mid p_{i}=\left(x_{i}, y_{i}\right)\right\}_{i=1}^{N}$. Now, we define the forward discrete difference operator as: $D^{+} p_{i}=p_{i+1}-p_{i}$, the central discrete difference operator as: $D^{c} p_{i}=\frac{p_{i+1}-p_{i-1}}{2}$, and the backward discrete difference operator as: $D^{-} p_{i}=p_{i}-p_{i-1}$.

\subsection{The Edge Detector Function}

Let $f$ be a given grayscale image, such that $f: \mathbb{R}^{2} \rightarrow \mathbb{R}^{1}$. That is, there is a corresponding pixel intensity for each given pair of coordinates in the domain of the image.

We now define $g(x, y \mid f)$ as an edge detector function that maps the change in pixel intensity of an image to a scalar value, such that $g: \mathbb{R}^{2} \rightarrow[0,1]$. This function must approach zero as the coordinates $(x, y)$ approach a non-homogenous region in the image, a perceived edge. Any function that exhibits similar behavior can be used in this model. We 
adopt the following the edge detector in our numerical implementation:

$$
g(x, y \mid f)=\frac{1}{1+a\left|D^{c}((k * f)(x, y))\right|^{p}}, p \geq 1,
$$

where $a$ and $p$ are tuning parameters, and $k$ is Gaussian kernel that is convolved (2-D) with the image $\mathrm{f}$ centered at $(x, y)$. Now let $I$ be the convolution of the filter and image at $(x, y)$ :

$$
I=(k * f)(x, y) \text {. }
$$

The result is a computable formulation of the main term of the edge detector involving the gradient as follows:

$$
\left|D^{c} I(x, y)\right|=\sqrt{\left(D_{x}^{c} I(x, y)\right)^{2}+\left(D_{y}^{c} I(x, y)\right)^{2}} .
$$

\subsection{Discrete Geodesic Active Contour}

We start by defining a continuous parametric curve $c(t)$ that is a counter clockwise representation of the boundary of a simple shape (i.e. closed set, no holes):

$$
C(t)=\{(x, y) \mid x=x(t), y=y(t), 0 \leq t \leq 1\}
$$

Then the continuous geodesic active contour energy is defined by the positively oriented line integral as follows:

$$
J[C]=\oint_{c} g(x, y) d s=\int_{a}^{b} g(C(t))\left|C^{\prime}(t)\right| d t .
$$

From here it is time to discretize the model. We define the continuous positively oriented curve $c$ to be equal to the set $P$ of vertices of a polygon of $N$ vertices,

$$
P=\left\{p_{i} \mid p_{i}=\left(x\left(t_{i}\right), y\left(t_{i}\right)\right)\right\}_{i=1}^{N}
$$

It is now easy to define the discrete line integral, as $P$ is positively oriented by definition. The discrete analogue is as follows:

$$
J[P]=\sum_{P} g(x, y) \Delta s=\sum_{i=1}^{N} g\left(p_{i}\right)\left|D^{+} p_{i}\right| \Delta i=\sum_{i=1}^{N} g\left(p_{i}\right)\left|D^{+} p_{i}\right|,
$$

we set $\Delta i=1$ as the indexing system is integer based. The positively oriented line integral becomes a sum over the $N$ vertices of the polygonal representation of the curve.

\subsection{Clique Based Shape Calculation}

We now consider a polygonal representation of a general closed region. First we define $r_{i}$ to be the $i$-th vertex of the polygon,

$$
r_{i}=\left(x_{i}^{r}, y_{i}^{r}\right) .
$$

Then let $S$ be the set of $N$ vertices that represent the polygon,

$$
S=\left\{r_{i}\right\}_{i=1}^{N} .
$$


It is now possible to define the intervertex distance between the $i$-th and $j$-th vertices of the general representation of a polygon:

$$
d_{i, j}=\left|r_{i}-r_{j}\right| .
$$

Then $\left[d_{i, j}\right]$ is a symmetric matrix, which holds all of the intervertex distances and is entirely dependent on the polygon being represented. We utilize a variant of the shape prior proposed by Elad and Kimmel [3,4]. This signature was previously incorporated into a piecewise-constant polygonal implementation of the Mumford Shah functional by Park. The model is based on the approximation of geometric distances with Euclidean distances in two dimensional space, we consider the intervertex distances of uniformly distributed points on the boundary of a two dimensional shape. It is known that the signature formed by the intervertex distances uniquely identify convex shapes, and has been applied to surface classification [15] and facial recognition problems [3, 4].

We now consider the polygonal representation of the curve $P(7)$ and compare its shape signature with that of a reference shape, denoted as $d_{i, j}$. The clique based shape term is now defined as:

$$
\inf _{P, s}\left\{\sum_{i, j=1}^{N}\left(\left|p_{i}-p_{j}\right|-s d_{i, j}^{2}\right)^{2}\right\} .
$$

Where $\left|p_{i}-p_{j}\right|$ is the distance between the $i$-th and the $j$-th vertex of the polygon, and the parameter $s$ is for scale invariance and can be obtained by minimization.

\subsection{Proposed Shape Prior Segmentation Model}

The proposed model is obtained by minimizing over both the geodesic active contour and the clique based shape prior energies simultaneously. The result is an optimization problem given by:

$$
\min _{P, s}\{\underbrace{\sum_{i=1}^{N} g\left(p_{i}\right)\left|D^{+} p_{i}\right|}_{J_{1}[P]}+\underbrace{\left.\alpha \sum_{i, j=1}^{N}\left(\left|p_{i}-p_{j}\right|^{2}-s d_{i, j}^{2}\right)\right\}}_{J_{2}[P]},
$$

where $\mathrm{P}$ is the evolving polygon, $\alpha$ is a shape parameter, $d_{i, j}$ is the $i$ - $j$-th intervertex distance of the reference shape, and $s$ is a scale parameter.

The main idea with the proposed model is to utilize shape information to boost the accuracy of the boundary approximations during segmentation.

\section{Energy Minimization}

The gradient descent algorithm will be used to minimize the non-convex energy over the set of vertices $\mathrm{P}$. Therefore we must find the velocity contribution of the energies in the descent direction.

\subsection{Geodesic Active Contour Variation}

In order to find the velocity contribution of the geodesic active contour term we compute the Gâteaux derivative to obtain the functional gradient of $J_{1}[P][31]$ as follows,

$$
\left.\frac{d}{d \epsilon} J_{1}[P+\epsilon V]\right|_{\epsilon=0}=\left\langle\nabla J_{1}[P], v\right\rangle \text {. }
$$


Expanding expression (14) yields:

$$
\left.\frac{d}{d \epsilon} J_{1}[P+\epsilon V]\right|_{\epsilon=0}=\left.\frac{d}{d \epsilon} \sum_{i=1}^{N} g\left(p_{i}+\epsilon v_{i}\right)\left|D^{+}\left(p_{i}+\epsilon v_{i}\right)\right|\right|_{\epsilon=0},
$$

where evaluation at $\epsilon=0$ will proceed after further transformation. Transforming the righthand side of the following:

$$
\begin{aligned}
& \sum_{i=1}^{N} \frac{d}{d \epsilon} g\left(p_{i}+\epsilon v_{i}\right)\left|D^{+}\left(p_{i}+\epsilon v_{i}\right)\right| \\
& =\sum_{i=1}^{N}\left(\frac{d}{d \epsilon} g\left(p_{i}+\epsilon v_{i}\right)\right)\left|D^{+}\left(p_{i}+\epsilon v_{i}\right)\right|+g\left(p_{i}+\epsilon v_{i}\right)\left(\frac{d}{d \epsilon}\left|D^{+}\left(p_{i}+\epsilon v_{i}\right)\right|\right) .
\end{aligned}
$$

Using the chain rule we obtain, $\frac{d}{d \epsilon} g\left(p_{i}+\epsilon v_{i}\right)=D^{c} g \cdot v$. Substituting into (16) gives:

$$
\begin{gathered}
\sum_{i=1}^{N}\left(D^{c} g\left(p_{i}+\epsilon v_{i}\right) \cdot v_{i}\right)\left|D^{+}\left(p_{i}+\epsilon v_{i}\right)\right|+g\left(p_{i}+\epsilon v_{i}\right) \frac{D^{+}\left(p_{i}+\epsilon v_{i}\right)}{\left|D^{+}\left(p_{i}+\epsilon v_{i}\right)\right|} \cdot \frac{d}{d \epsilon}\left(D^{+}\left(p_{i}+\epsilon v_{i}\right)\right) \\
=\sum_{i=1}^{N}\left(D^{c} g\left(p_{i}+\epsilon v_{i}\right) \cdot v_{i}\right)\left|D^{+}\left(p_{i}+\epsilon v_{i}\right)\right|+g\left(p_{i}+\epsilon v_{i}\right) \frac{D^{+}\left(p_{i}+\epsilon v_{i}\right)}{\left|D^{+}\left(p_{i}+\epsilon v_{i}\right)\right|} \cdot D^{+} v_{i} .
\end{gathered}
$$

Now evaluating (17) at $\epsilon=0$ results in,

$$
\frac{d}{d \epsilon} J_{1}[P]=\sum_{i=1}^{N}\left(D^{c} g\left(p_{i}\right) \cdot v_{i}\right)\left|D^{+} p_{i}\right|+g\left(p_{i}\right) \frac{D^{+} p_{i}}{\left|D^{+} p_{i}\right|} \cdot D^{+} v_{i} .
$$

We now use summation by parts, the right hand side of equation (18) becomes:

$$
g\left(p_{n}\right) \frac{D^{+} p_{n}}{\left|D^{+} p_{n}\right|} \cdot v_{n+1}-g\left(p_{1}\right) \frac{D^{+} p_{1}}{\left|D^{+} p_{1}\right|} \cdot v_{1}+\sum_{i=1}^{N}\left(D^{c} g\left(p_{i}\right) \cdot v_{i}\right)\left|D^{+} p_{i}\right|-D^{-}\left(g\left(p_{i}\right) \frac{D^{+} p_{i}}{\left|D^{+} p_{i}\right|}\right) \cdot v_{i} .
$$

For some $\gamma, \zeta \in \mathbb{R}^{2}$, we have that $p_{1}=\gamma$ and $p_{n}=\zeta$. In addition, $p_{1}=p_{n+1}$. The varied function $\hat{P}=P+\varepsilon V$ must satisfy the same conditions as $P$ on its boundary, that is $\hat{p_{1}}=p_{1}+\varepsilon v_{1}=\gamma$ and $\hat{p_{n}}=p_{n}+\varepsilon v_{n}=\zeta$, therefore $v_{1}=0$ and $v_{n}=0$. Thus $v_{1}=v_{n+1}=0$ and (19) becomes:

$$
\sum_{i=1}^{N}\left(D^{c} g\left(p_{i}\right) \cdot v_{i}\right)\left|D^{+} p_{i}\right|-D^{-}\left(g\left(p_{i}\right) \frac{D^{+} p_{i}}{\left|D^{+} p_{i}\right|}\right) \cdot v_{i} .
$$

Via the chain rule, $D^{-} g\left(p_{i}\right)=\frac{\partial g}{\partial x} D^{-} x_{i}+\frac{\partial g}{\partial y} D^{-} y_{i}=D^{c} g\left(p_{i}\right) \cdot D^{-} p_{i}$, substituting gives:

$$
\sum_{i=1}^{N} D^{c} g\left(p_{i}\right) \cdot v_{i}\left|D^{+} p_{i}\right|-\left(D^{c} g\left(p_{i}\right) \cdot D^{-} p_{i} \frac{D^{+} p_{i}}{\left|D^{+} p_{i}\right|}+g\left(p_{i}\right) D^{-} \frac{D^{+} p_{i}}{\left|D^{+} p_{i}\right|}\right) \cdot v_{i} .
$$


The discrete unit tangent can be formulated as $T_{i}=\frac{D^{+} p_{i}}{\left|D^{+} p_{i}\right|}$, this yields:

$$
\sum_{i=1}^{N}\left(D^{c} g\left(p_{i}\right)\left|D^{+} p_{i}\right|-D^{c} g\left(p_{i}\right) \cdot D^{-} p_{i} T_{i}-g\left(p_{i}\right) D^{-} T_{i}\right) \cdot v_{i}
$$

Now, assuming convergence of the discrete model to the continuous one, we can set $T_{i}=$ $\frac{D^{-} T_{i}}{\left|D^{+} p_{i}\right|}$. We then use the following $D^{-} p_{i}=\frac{D^{-} p_{i}}{\left|D^{+} p_{i}\right|}\left|D^{+} p_{i}\right|=T_{i}\left|D^{+} p_{i}\right|$ and $D^{-} T_{i}=\frac{D^{-} T_{i}}{\left|D^{+} p_{i}\right|}\left|D^{+} p_{i}\right|$ to rewrite $(22)$ in more familiar geometric terms,

$$
\begin{aligned}
& \sum_{i=1}^{N}\left(D^{c} g\left(p_{i}\right)\left|D^{+} p_{i}\right|-D^{c} g\left(p_{i}\right) \cdot T_{i}\left|D^{+} p_{i}\right| T_{i}-g\left(p_{i}\right) \frac{D^{-} T_{i}}{\left|D^{+} p_{i}\right|}\left|D^{+} p_{i}\right|\right) \cdot v_{i} \\
& =\sum_{i=1}^{N}\left[\left(D^{c} g\left(p_{i}\right)-\left(D^{c} g\left(p_{i}\right) \cdot T_{i}\right) T_{i}-g\left(p_{i}\right) \frac{D^{-} T_{i}}{\left|D^{+} p_{i}\right|}\right) \cdot v_{i}\right]\left|D^{+} p_{i}\right| .
\end{aligned}
$$

Simplification is obtained via vector orthogonality, i.e.

$D^{c} g\left(p_{i}\right)-\left(D^{c} g\left(p_{i}\right) \cdot T_{i}\right) T_{i}=\left(D^{c} g\left(p_{i}\right) \cdot N_{i}\right) N_{i}$. Further yet, $\left(D^{c} g\left(p_{i}\right) \cdot N_{i}\right) N_{i}=D_{N_{i}}^{c} g\left(p_{i}\right) N_{i}$, where $D_{N_{i}}^{c} g\left(p_{i}\right)$ is the directional derivative. These simplifications yield:

$$
\sum_{P}\left[\left(D_{N_{i}}^{c} g\left(p_{i}\right) N_{i}-g\left(p_{i}\right) \frac{D^{-} T_{i}}{\left|D^{+} p_{i}\right|}\right) \cdot v_{i}\right]\left|D^{+} p_{i}\right|
$$

We then change the bounds of discrete integration, as we know that $\Delta s_{i}=\left|D^{+} p_{i}\right|$,

$$
\sum_{P}\left[\left(D_{N_{i}}^{c} g\left(p_{i}\right) N_{i}-g\left(p_{i}\right) \frac{D^{-} T_{i}}{\left|D^{+} p_{i}\right|}\right) \cdot v_{i}\right] \Delta s_{i}
$$

Now use the following discrete analogues to well known definitions from differential geometry to construct an intuitive formulation of the above equation: $\kappa_{i}=\frac{\left|D^{-} T_{i}\right|}{\left|D^{+} p_{i}\right|}, N_{i}=\frac{\frac{D^{-} T_{i}}{\left|D^{+} p_{i}\right|}}{\left|\frac{D^{-} T_{i}}{\left|D^{+} p_{i}\right|}\right|}$ and $\frac{D^{-} T_{i}}{\left|D^{+} p_{i}\right|}=\kappa_{i} N_{i}$. Where $k$ is the curvature and $N$ is the unit normal vector. Substitution yields:

$$
\sum_{P}\left[\left(D_{N_{i}}^{c} g\left(p_{i}\right) N_{i}-g\left(p_{i}\right) \kappa_{i} N_{i}\right) \cdot v_{i}\right] \Delta s_{i}=0 .
$$

The functional gradient is recovered from (26), for all variations of $v$ represented by $\left\{v_{i}\right\}_{i=1}^{N}$ in the following form:

$$
\frac{d}{d \epsilon} J_{1}[P]=\left\langle(\underbrace{\left.D_{N_{i}}^{c} g\left(p_{i}\right)-g\left(p_{i}\right) \kappa_{i}\right) N_{i}}_{\text {functional gradient }}, v_{i}\rangle .\right.
$$

The velocity contribution of the geodesic active contour term in gradient descent is given by the following:

$$
v_{i}^{G A C}=-\left(D_{N_{i}}^{c} g\left(p_{i}\right)-g\left(p_{i}\right) \kappa_{i}\right) N_{i}
$$

Note that this velocity is parallel to the gradient descent direction, and will therefore contribute all of its magnitude (i.e. $v_{i}^{G A C}=\operatorname{proj}_{N_{i}} v_{i}^{G A C}$ ). Therefore:

$$
{\dot{p_{i}}}^{G A C}=-\left(D_{N_{i}}^{c} g\left(p_{i}\right)-g\left(p_{i}\right) \kappa_{i}\right) N_{i} .
$$




\subsection{Clique Shape Signature Variation}

Now it is time to solve for the velocity contribution of the shape term:

$$
J_{2}[P]=\alpha \sum_{i, j=1}^{N}\left(\left|p_{i}-p_{j}\right|^{2}-s d_{i, j}^{2}\right)^{2}
$$

In order to formulate the Gâteaux derivative of the $k$-th vertex we fix $i=k$, this allows for a simple calculation that yields the velocity contribution of the $k$-th vertex independently i.e. $J_{2}\left[p_{k}\right]$. Consequently, this reduces the double sum to a single sum:

$$
\left.\frac{d}{d \epsilon} J_{2}[P+\epsilon V]\right|_{\epsilon=0}=\left.\alpha \sum_{j=1}^{N} \frac{d}{d \epsilon}\left(\left|\left(p_{k}+\epsilon v_{k}\right)-p_{j}\right|^{2}-s d_{k, j}^{2}\right)^{2}\right|_{\epsilon=0}
$$

In turn, the right hand side of (31) evaluates to:

$$
\begin{aligned}
& 2 \alpha \sum_{j=1}^{N}\left(\left|\left(p_{k}+\epsilon v_{k}\right)-p_{j}\right|^{2}-s d_{k, j}^{2}\right) \frac{d}{d \epsilon}\left(\left|\left(p_{k}+\epsilon v_{k}\right)-p_{j}\right|^{2}-s d_{k, j}^{2}\right) \\
& =4 \alpha \sum_{j=1}^{N}\left(\left|\left(p_{k}+\epsilon v_{k}\right)-p_{j}\right|^{2}-s d_{k, j}^{2}\right)\left|\left(p_{k}+\epsilon v_{k}\right)-p_{j}\right| \frac{d}{d \epsilon}\left|\left(p_{k}+\epsilon v_{k}\right)-p_{j}\right| \\
& =4 \alpha \sum_{j=1}^{N}\left(\left|\left(p_{k}+\epsilon v_{k}\right)-p_{j}\right|^{2}-s d_{k, j}^{2}\right)\left|\left(p_{k}+\epsilon v_{k}\right)-p_{j}\right| \\
& \frac{\left(p_{k}+\epsilon v_{k}\right)-p_{j}}{\left|\left(p_{k}+\epsilon v_{k}\right)-p_{j}\right|} \cdot \frac{d}{d \epsilon}\left(\left(p_{k}+\epsilon v_{k}\right)-p_{j}\right) \\
& =4 \alpha \sum_{j=1}^{N}\left(\left|\left(p_{k}+\epsilon v_{k}\right)-p_{j}\right|^{2}-s d_{k, j}^{2}\right)\left(\left(p_{k}+\epsilon v_{k}\right)-p_{j}\right) \cdot v_{k} .
\end{aligned}
$$

Now, take the limit as $\epsilon$ goes to zero of (32) and drop the constant:

$$
\alpha \sum_{j=1}^{N}\left(\left|p_{k}-p_{j}\right|^{2}-s d_{k, j}^{2}\right)\left(p_{k}-p_{j}\right) \cdot v_{k}
$$

Then we rewrite (33) as the following,

$$
\frac{d}{d \epsilon} J_{2}[P]=\underbrace{\left\langle\alpha \sum_{j=1}^{N}\left(\left|p_{k}-p_{j}\right|^{2}-s d_{k, j}^{2}\right)\left(p_{k}-p_{j}\right)\right.}_{\text {functional gradient }}, v_{k}\rangle .
$$

The shape prior term yields the following velocity contribution to the gradient descent:

$$
v_{k}^{S P}=-\alpha \sum_{j=1}^{N}\left(\left|p_{k}-p_{j}\right|^{2}-s d_{k, j}^{2}\right)\left(p_{k}-p_{j}\right) .
$$


We now project the velocity (35) in the inward normal direction, as this is well known to be the direction that will minimize arc length, in essence we replace curvature with shape information

$$
{\dot{p_{i}}}^{S P}=-\alpha\left[\left(\sum_{j=1}^{N}\left(\left|p_{i}-p_{j}\right|^{2}-s d_{i, j}^{2}\right)\left(p_{i}-p_{j}\right)\right) \cdot N_{i}\right] N_{i} .
$$

Optimization with respect to the scale parameters leads to the following condition [34]:

$$
s=\frac{\sum_{i, j}\left|p_{i}-p_{j}\right|^{2} d_{i, j}^{2}}{\sum_{i, j} d_{i, j}^{4}}
$$

\subsection{The Functional Gradient}

The combination of both terms, gives us the following ODE system on the vertices of the polygon,

$$
\dot{p}_{i}=-\left(D_{N_{i}}^{c} g\left(p_{i}\right)-g\left(p_{i}\right) \kappa_{i}\right) N_{i}-\alpha\left[\left(\sum_{j=1}^{N}\left(\left|p_{i}-p_{j}\right|^{2}-s d_{i, j}^{2}\right)\left(p_{i}-p_{j}\right)\right) \cdot N_{i}\right] N_{i} .
$$

In the discrete time setting,

$$
\frac{p_{i}^{n+1}-p_{i}^{n}}{\Delta t}=-\left(D_{N_{i}}^{c} g\left(p_{i}\right)-g\left(p_{i}\right) \kappa_{i}\right) N_{i}-\alpha\left[\left(\sum_{j=1}^{N}\left(\left|p_{i}-p_{j}\right|^{2}-s d_{i, j}^{2}\right)\left(p_{i}-p_{j}\right)\right) \cdot N_{i}\right] N_{i} .
$$

We arrive at the gradient descent algorithm in a time dependent form:

$$
\begin{aligned}
& p_{i}^{n+1}=p_{i}^{n}+\Delta t\left[-\left(D_{N_{i}^{n}}^{c} g\left(p_{i}^{n}\right)-g\left(p_{i}^{n}\right) \kappa_{i}^{n}\right)\right. \\
& \left.-\alpha\left[\left(\sum_{j=1}^{N}\left(\left|p_{i}^{n}-p_{j}^{n}\right|^{2}-s^{n} d_{i, j}^{2}\right)\left(p_{i}^{n}-p_{j}^{n}\right)\right) \cdot N_{i}^{n}\right]\right] N_{i}^{n} .
\end{aligned}
$$

\section{Numerical Implementation}

An overview of the proposed algorithm follows below.

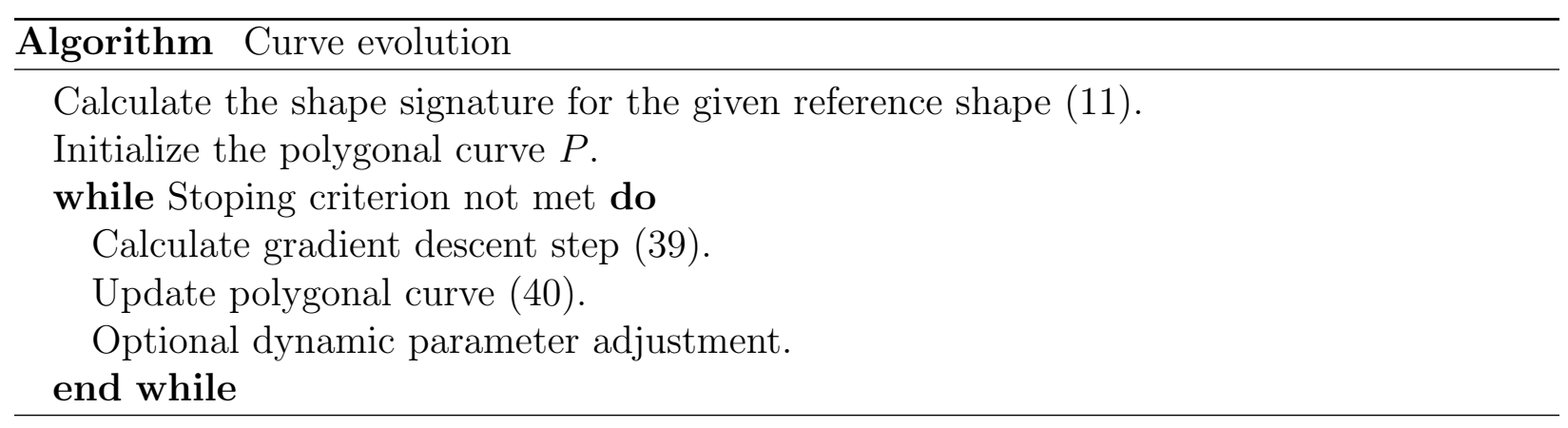


We define the stopping criterion to be $\left|p^{n+1}-p^{n}\right|<\epsilon$, for an appropriately chosen $\epsilon$. A more accurate fit can be attained at the cost of additional vertices in the parameterization.

It is important to note that the initialization of the polygonal curve $P$ has the potential to alter the minimization. The best results are generally found when the initial parameterization encloses the desired object as curve evolution is generally in the inward normal direction.

\subsection{Edge Detector Implementation}

The edge detector function $g(x, y \mid f)$ is parameterized by three tuning parameters $a, p$, and $k$ (2). The parameters $a$ and $p$ are scalar-valued parameters that effect the strength of the edge detector. Throughout our experiments we found that $a=8$ and $p=2$ work well and kept them fixed at these values. The parameter $k$ is a discrete Gaussian filter that behaves as a point spread function when convolved with the given image. This operation removes small details and noise, effectively smoothing the image. The amount of smoothing is determined by its standard deviation $\sigma$. In our implementation, as standard, $k$ is an odd $n \times n$ matrix that represents a 2-D Gaussian distribution by holding sampled points of the continuous version centered at the center point of $k$ :

$$
k(i, j)=\frac{k_{g}(i, j)}{\sum_{i} \sum_{j} k_{g}}, \text { where } k_{g}(i, j)=e^{\frac{-\left(i^{2}+j^{2}\right)}{2 \sigma^{2}}} .
$$

\subsection{Model Implementation}

Direct computation of the geodesic active contour contribution to the gradient descent algorithm follows from the definitions in both the preliminary notation and the derivations (29).

The calculations associated with clique shape signature introduce a small amount of complexity to which we provide an elegant solution. The shape term (12) is the infimum of the energy calculation over every possible parameterization of the polygon $P$. This means that the shape velocity contribution (36) is given by the parameterization with the lowest energy, thus the model requires a built in auto-parameterization shift. To remain practical about computational complexity in our implementation we restrict the number of shifts in the parameterization that were tested. In our experiments we tested every 3rd reparameterization.

Our implementation follows a general shifting pattern over a predetermined set of integers: we make the $i$-th vertex the $i+n$-th vertex and compute the energy. The parameterization that results in the lowest energy is kept and gradient descent contribution is calculated with respect to the new parameterization. During this computation we check the energy the same number of shifts backwards as forwards. The algorithm described above is shown as follows. This algorithm is computed during each step of gradient descent, and the actual parameterization of the curve is shifted by the $n$ that gives the lowest energy. We note that $d_{i, j}$, the reference shape, is only calculated once prior to entering gradient descent.

\subsection{Time Dependent Parameters}

Throughout our numerical experiments we found that it is possible to achieve better performance if we treat some of the parameters namely $\alpha$ and $\sigma$ as functions of discrete time. This allows the model to vary the influence of the shape parameter, the quantity of noise, and relative size of image features as a function of time. 


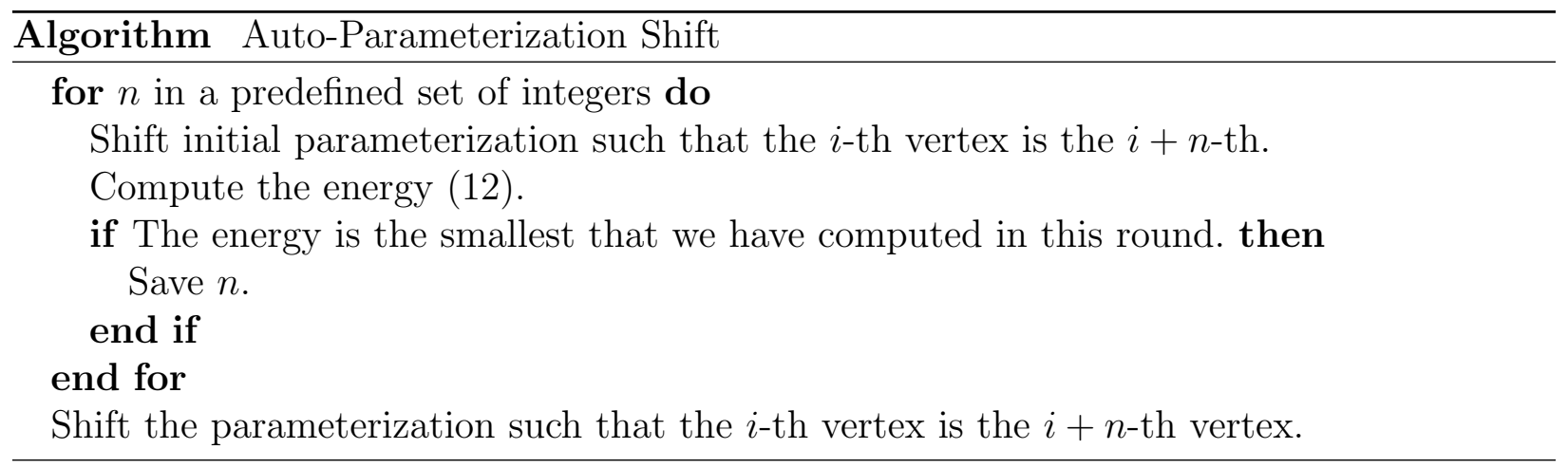

The influence of the shape energy on curve evolution is proportional to $\alpha$. Thus, an increasing $\alpha$ will let the model evolve based on image features before strictly enforcing the shape information.

Evolution of $\sigma$ alters the quantity of noise and image features in the model. We propose starting with a relatively large $\sigma$ and model its evolution as a decreasing function of time. This refinement technique allows the curve to localize better, as the edge detector itself becomes more localized over time. This techniques is particularly useful for illusory contours where partial edges contribute to data fidelity, and for noisy or cluttered images where pixel intensity variation can adversely effect curve evolution. These techniques mitigate some of the troubles that arise when the minimization forces the curve into a local minima.

\section{Experiments}

\subsection{Parameter Tuning}

The following numerical experiments utilized a scheme with a fixed time step, and variable shape velocity contribution $(\alpha)$, and variable standard deviation $(\sigma)$.

We experimentally determined that $\Delta t \in(0,0.5)$ result in convergence of the model, and thus chose our time step as large as possible to speed up convergence, thus we set $\Delta t=0.4$ throughout all experiments. This parameter could also possibly benefit from dynamic scheduling, as small $\Delta t$ could potentially allow for more refinement. The shape velocity contribution and standard deviation of the Gaussian filter were varied according to their own schedules with synchronous update. Throughout all of our expierments we found that $\sigma(t) \in[0.001,60]$ and $\alpha(t) \in\left[0,10^{-9}\right]$ generally work well with the model. We evolved $\alpha$ on an exponential growth schedule and $\sigma$ on a linear decay schedule. The schedulers involved consisted of setting $\alpha(t)$ to increasing powers of 10, where the powers are linearly spaced, with in its specified interval, and setting $\sigma$ to linearly spaced decreasing points within its specified interval. The above schedulers can be seen as a sequence of points sampled from $g(x)=10^{x}$ and $h(x)=x$. The explicit parameters used in each experiment are further detailed below.

\subsection{Illusory Contour Example}

An illusory contour is an optical illusion that elicits the perception of an edge without a color or illuminance change across said perceived edge. The absence of a homogenous region makes using region based segmentation methods and edge based segmentation methods 
difficult. The standard geodesic active contour model has difficulty moving out of local minima as demonstrated in Fig. 2. Throughout the following expierments in this section we let $\alpha \in\left[10^{-12}, 10^{-9}\right]$ and $\sigma \in[1,20]$.

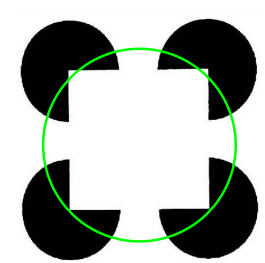

(a) Initialization without shape.

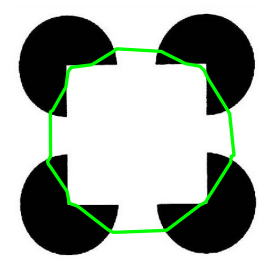

(c) Evolving

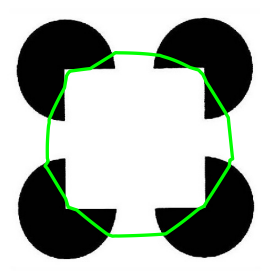

(b) Evolving

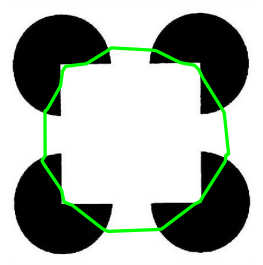

(d) Result

Figure 2: Standard geodesic active contour. Without shape information the model gets stuck in a local minima and the illusory contour is not captured.

Successful capture of illusory contours is demonstrated in Fig. 3, Fig. 4, and Fig. 5. 


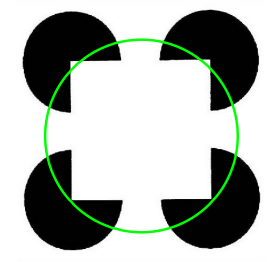

(a) Initialization

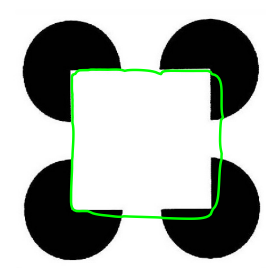

(c) Evolving

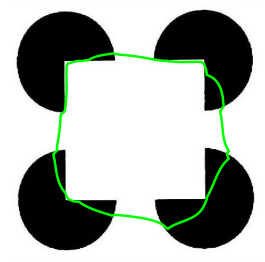

(b) Evolving

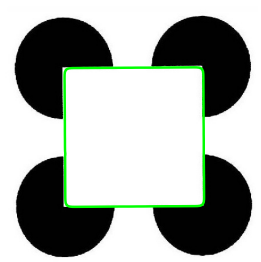

(d) Result

Figure 3: Illusory contour captured with aide of shape prior. Shape prior information allows the model to move out of undesired local minima and capture the illusory contour.

The combined contributions of $J_{1}[P]$ and $J_{2}[P]$ allow the model to perceive edges that do not exist and more easily assume the correct shape.

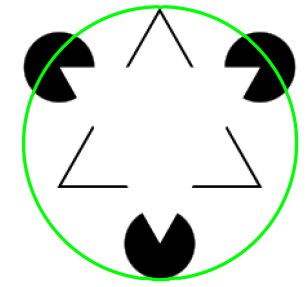

(a) Initialization

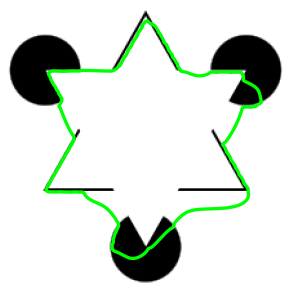

(c) Evolving

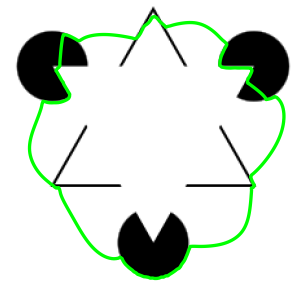

(b) Evolving

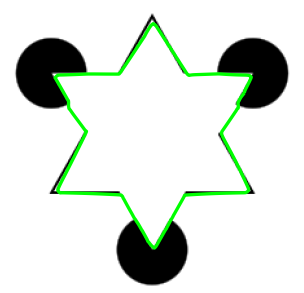

(d) Result

Figure 4: A less trivial example. An example of an illusory contour with sparse representation. Three of the corners are represented with simple line segments. 


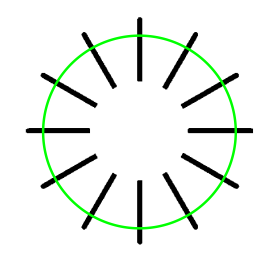

(a) Initialization

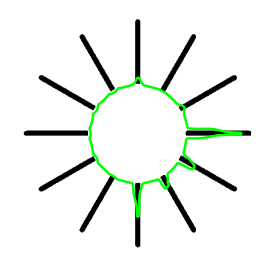

(c) Evolving

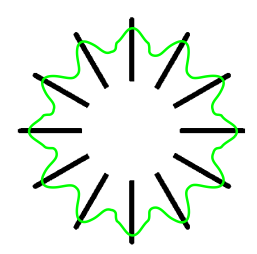

(b) Evolving

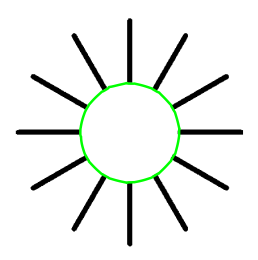

(d) Result

Figure 5: Successful segmentation. Another example of an illusory contour with sparse representation. The evolving curve is able to recover the curvature of the illusory contour.

\subsection{Cluttered Image Example}

A caveat of the standard geodesic active contour model, is that it must be localized prior to segmentation as it has a tendency to stick to clutter, as demonstrated in Fig.6. 


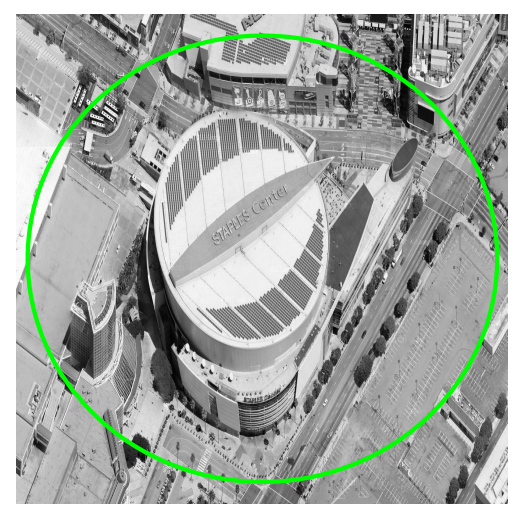

(a) Initialization without shape.

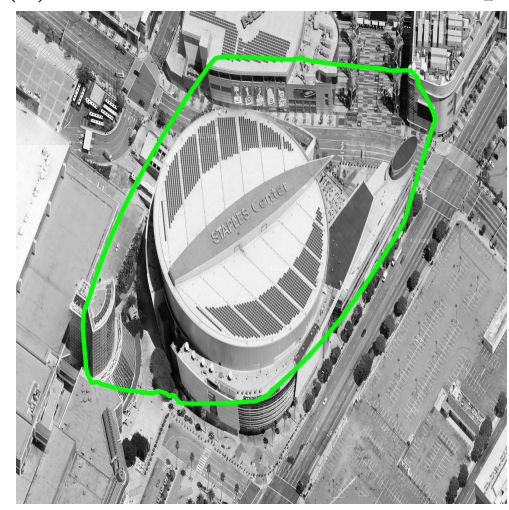

(c) Evolving

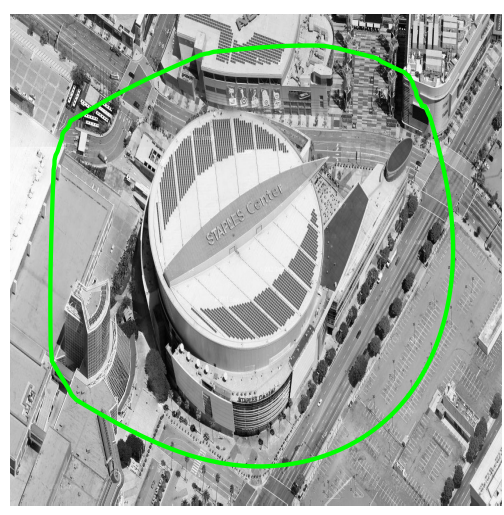

(b) Evolving

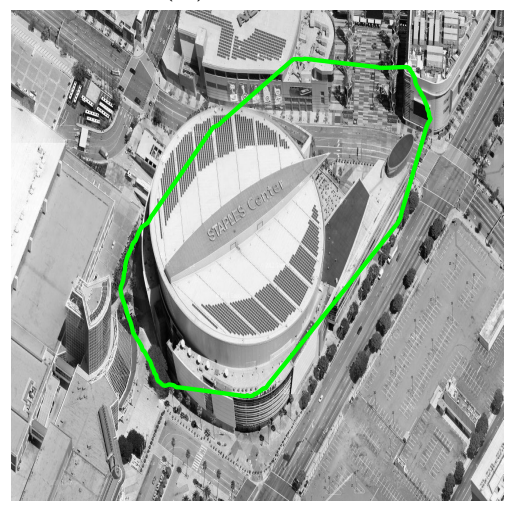

(d) Result

Figure 6: Segmentation of object in clutter without shape. Pixel intensity variation makes it difficult for the standard geodesic active to move out of local minima.

Our improvements on the model give it an increased ability to capture shapes in cluttered backgrounds. The evolving Gaussian filter allows the model to ignore variations in pixel intensity during the early stages of evolution. This allows the curve to localize itself in a way that optimally minimizes both $J_{1}[P]$ and $J_{2}[P]$ while $\sigma$ is large. As $\sigma$ decreases the curve is then able to shrink-wrap the boundary of the object. While this is happening the shape information guides the curve to resemble the reference shape. The combination of these motions allow for a much closer fit as seen in Fig.7 where the curve overcomes large variation in pixel intensity. We let $\alpha \in\left[10^{-30}, 10^{-9}\right]$ and $\sigma \in[1,60]$. 


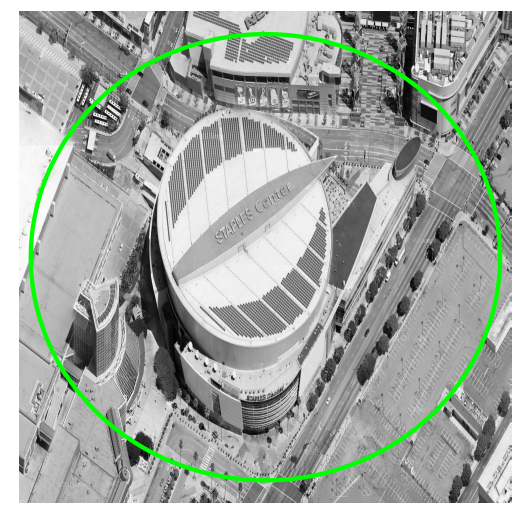

(a) Initialization

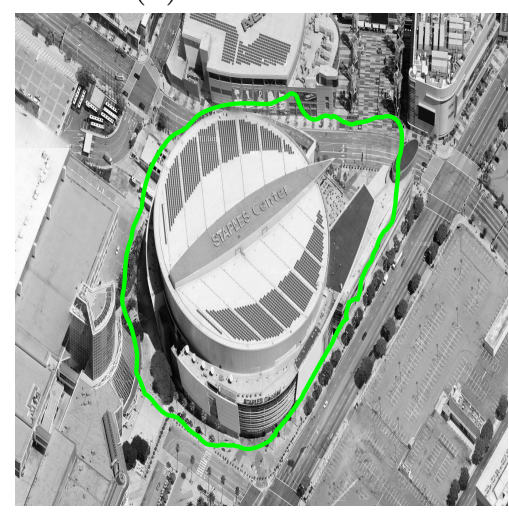

(c) Evolving

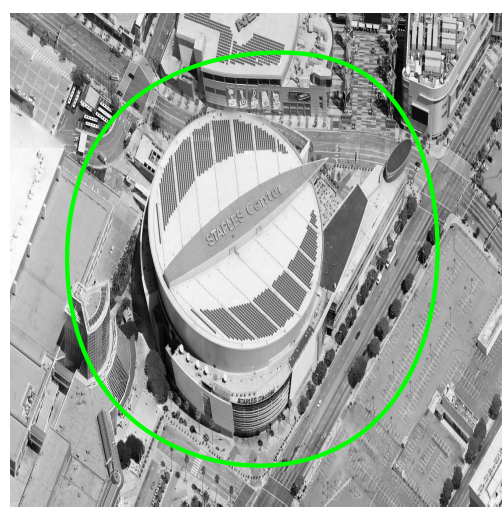

(b) Evolving

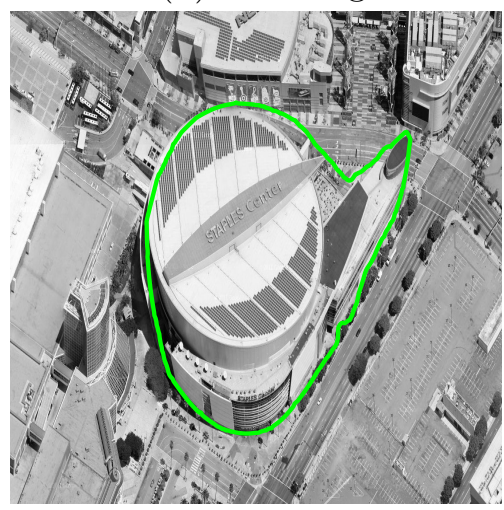

(d) Result

Figure 7: Segmentation of object in clutter with shape. Shape prior information combined with shrinking Gaussian support allows the model to move away from suboptimal solutions.

\subsection{Occluded Object Example}

The improved model is very suitable for occluded image segmentation where the location and size of the occluder are known prior to segmentation. This allows for explicit incorporation of global information into the model. A simple way of doing this is through the use of a mask that disables the velocity contribution of $J_{1}[P]$ for vertices that are on the occluder. We let $\alpha \in\left[0,10^{-9}\right]$ and fixed $\sigma=0.1$, the parameter evolution served a decreased role in segmentation. Evolving $\alpha$ in this situation is still desirable as it allows the edge based information to guide the model before shape contributes to curve evolution.

We define a set of points $M$ that covers the occluder.

$$
M=\{(x, y) \mid(x, y) \text { occludes the image }\}
$$

We can then define a function that will incorporate the mask into the model:

$$
f^{\text {mask }}(x, y)=\left\{\begin{array}{ll}
0 & (x, y) \in M \\
1 & (x, y) \notin M
\end{array}\right\} .
$$

Then the gradient descent update becomes:

$$
p_{i}^{n+1}=p_{i}^{n}+\Delta t\left[f^{\text {mask }}\left(p_{i}^{n}\right) \dot{p}_{i}^{G A C}+{\dot{p_{i}}}^{S P}\right] .
$$


An artificial example is given in Fig. 8, were an occluder is modeled by a gray square and the mask is the exact shape of the occluder.
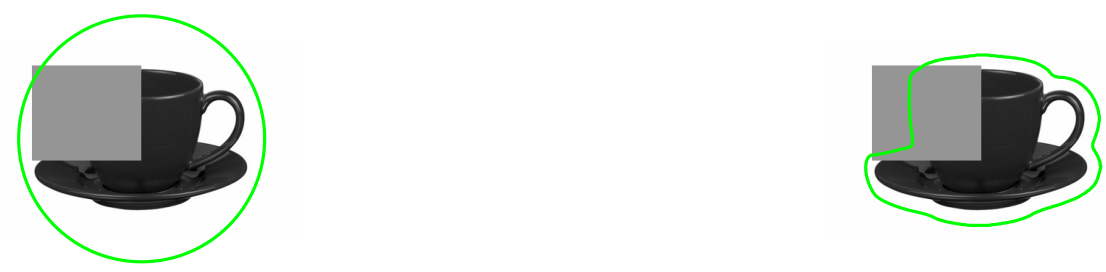

(a) Initialization

(b) Evolving
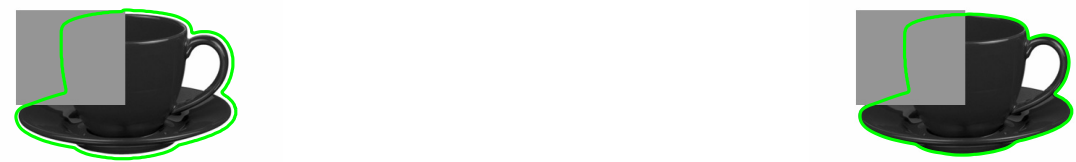

(c) Evolving

(d) Result

Figure 8: Image disocclusion. Despite significant missing information, the boundary of a stunning teacup is preserved.

Full recovery of an occluded region on a natural image is demonstrated in Fig. 9. In this experiment we let $\alpha \in\left[0,10^{-9}\right]$ and $\sigma \in[1.3,30]$. 


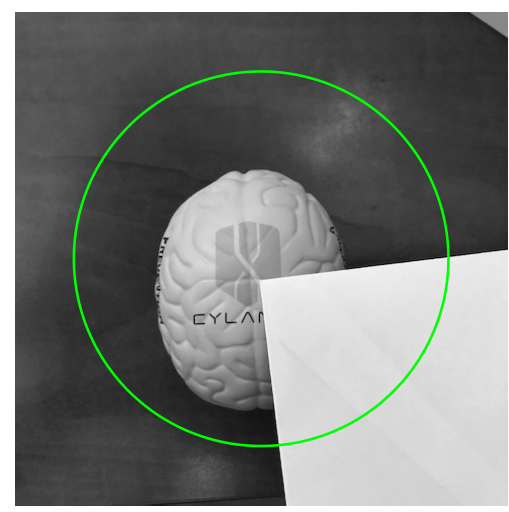

(a) Initialization

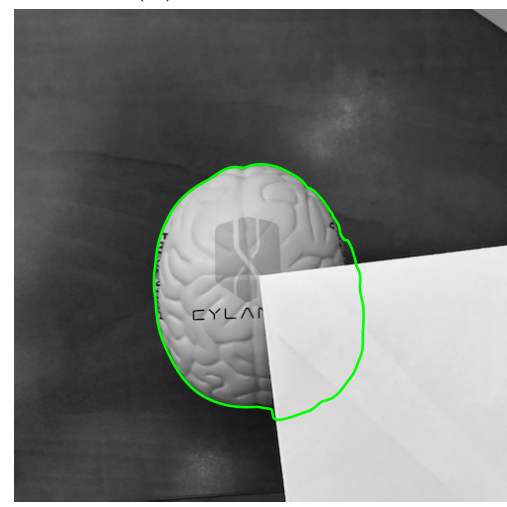

(c) Evolving

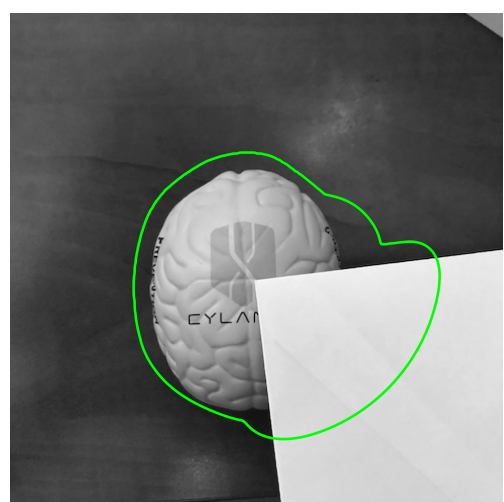

(b) Evolving

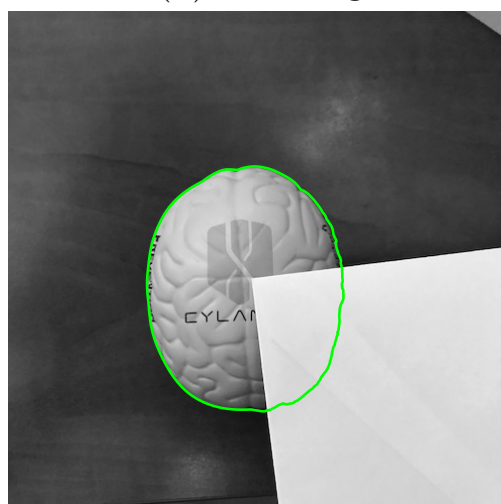

(d) Result

Figure 9: Disocclusion real example. Shape information allows us to capture a feature that is occluded.

\section{Conclusions and Future Work}

We have explicitly derived a discrete implementation of the geodesic active contour model and incorporated a clique based shape prior energy. The evolving Gaussian refinement technique was proposed, and the parameterization shifting algorithm was explicitly laid out. Both techniques are automated in the model. The numerical results demonstrated the models ability to accurately segment illusory contours, objects in cluttered backgrounds, and occluded objects.

Future work involves applying machine learning techniques to boost the performance of the model. 


\section{References}

[1] X. Bresson, S. Esedoglu, P. Vandergheynst, J.-P. Thiran, and S. Osher, Fast global minimization of the active contour/snake model, J. Math. Imaging Vis., 28 (2007), pp. 151-167.

[2] X. Bresson, P. Vandergheynst, and J.-P. Thiran, A variational model for object segmentation using boundary information and shape prior driven by the mumford-shah functional, Int. J. Comput. Vision, 68 (2006), pp. 145-162.

[3] A. M. Bronstein, M. M. Bronstein, And R. Kimmel, Three-dimensional face recognition, International Journal of Computer Vision, 64 (2005), pp. 5-30.

[4] A. M. Bronstein, M. M. Bronstein, and R. Kimmel, Expression-invariant representations of faces, Trans. Img. Proc., 16 (2007), pp. 188-197.

[5] E. S. Brown, T. F. Chan, and X. Bresson, Completely convex formulation of the chan-vese image segmentation model, Int. J. Comput. Vision, 98 (2012), pp. 103-121.

[6] V. Caselles, R. Kimmel, and G. Sapiro, Geodesic active contours, Int. J. Comput. Vision, 22 (1997), pp. 61-79.

[7] T. Chan And W. Zhu, Level set based shape prior segmentation, in Proceedings of the 2005 IEEE Computer Society Conference on Computer Vision and Pattern Recognition (CVPR'05) - Volume 2 - Volume 02, CVPR '05, Washington, DC, USA, 2005, IEEE Computer Society, pp. 1164-1170.

[8] T. F. Chan And L. A. Vese, Active contours without edges, Trans. Img. Proc., 10 (2001), pp. 266-277.

[9] C. Chesnaud, P. RéfréGier, And V. Boulet, Statistical region snake-based segmentation adapted to different physical noise models, IEEE Trans. Pattern Anal. Mach. Intell., 21 (1999), pp. 1145-1157.

[10] D. Cremers, T. Kohlberger, And C. Schnörr, Nonlinear shape statistics in mumford-shah based segmentation, in Proceedings of the 7th European Conference on Computer Vision-Part II, ECCV '02, London, UK, UK, 2002, Springer-Verlag, pp. 93108.

[11] E. Debreuve, M. Gastaud, M. Barlaud, and G. Aubert, Using the shape gradient for active contour segmentation: From the continuous to the discrete formulation, J. Math. Imaging Vis., 28 (2007), pp. 47-66.

[12] S. Derrode, M. A. Charmi, and F. Ghorbel, Fourier-based invariant shape prior for snakes, in Acoustics, Speech and Signal Processing, 2006. ICASSP 2006 Proceedings. 2006 IEEE International Conference on, vol. 2, IEEE, 2006, pp. II-II. 
[13] B. Dong AND Z. Shen, Mra-based wavelet frames and applications: Image segmentation and surface reconstruction, in Independent Component Analyses, Compressive Sampling, Wavelets, Neural Net, Biosystems, and Nanoengineering X, vol. 8401, International Society for Optics and Photonics, 2012, p. 840102.

[14] G. DoĞan, P. Morin, And R. H. Nochetto, A variational shape optimization approach for image segmentation with a mumford-shah functional, SIAM J. Sci. Comput., 30 (2008), pp. 3028-3049.

[15] A. Elad And R. Kimmel, On bending invariant signatures for surfaces, IEEE Trans. Pattern Anal. Mach. Intell., 25 (2003), pp. 1285-1295.

[16] A. Foulonneau, P. Charbonnier, and F. Heitz, Affine-invariant geometric shape priors for region-based active contours, IEEE Trans. Pattern Anal. Mach. Intell., 28 (2006), pp. 1352-1357.

[17] F. Galland, A. Jaegler, M. Allain, D. Savéry, and P. RÉfrégier, Smooth contour coding with minimal description length active grid segmentation techniques, Pattern Recogn. Lett., 32 (2011), pp. 721-730.

[18] M. Gastaud, M. Barlaud, and G. Aubert, Combining shape prior and statistical features for active contour segmentation, IEEE TRANS. CIRCUITS SYST. VIDEO TECHNOL, 14 (2004), pp. 726-734.

[19] L. Jonasson, X. Bresson, P. Hagmann, O. Cuisenaire, R. Meuli, and J.-P. THIRAN, White matter fiber tract segmentation in dt-mri using geometric flows, Medical Image Analysis, 9 (2005), pp. 223-236.

[20] Y. M. JUng AND J. J. SHEN, Illusory shapes via first-order phase transition and approximation, Journal of Mathematical Imaging and Vision, 53 (2015), pp. 303-313.

[21] S. H. Kang, W. Zhu, And J. JiAnhong, Illusory shapes via corner fusion, SIAM Journal on Imaging Sciences, 7 (2014), pp. 1907-1936.

[22] G. Kanizsa and G. Kanizsa, Organization in vision: Essays on Gestalt perception, vol. 49, Praeger New York, 1979.

[23] M. Kass, A. Witkin, And D. Terzopoulos, Snakes: Active contour models, INTERNATIONAL JOURNAL OF COMPUTER VISION, 1 (1988), pp. 321-331.

[24] S. Kichenassamy, A. Kumar, And P. Olver, Conformal curvature flows: From phase transitions to active vision, 1995.

[25] V. Kolmogorov And R. ZABIH, Multi-camera scene reconstruction via graph cuts, in European conference on computer vision, Springer, 2002, pp. 82-96.

[26] - What energy functions can be minimized via graph cuts?, in European conference on computer vision, Springer, 2002, pp. 65-81. 
[27] J. Liang, F. PARK, AND H. ZhaO, Robust and efficient implicit surface reconstruction for point clouds based on convexified image segmentation, Journal of Scientific Computing, 54 (2013), pp. 577-602.

[28] S. LuO AND X.-C. TAI, Convex shape priors for level set representation, arXiv preprint arXiv:1811.04715, (2018).

[29] R. Malladi, R. Kimmel, D. Adalsteinsson, G. Sapiro, V. Caselles, and J. A. Sethian, A geometric approach to segmentation and analysis of $3 d$ medical images, in Proceedings of the 1996 Workshop on Mathematical Methods in Biomedical Image Analysis (MMBIA '96), MMBIA '96, Washington, DC, USA, 1996, IEEE Computer Society, pp. 244-.

[30] D. Mumford and J. Shah, Optimal approximations by piecewise smooth functions and associated variational problems, Communications on pure and applied mathematics, 42 (1989), pp. 577-685.

[31] P. J. Olver, Introduction to the calculus of variations, University of Minnesota, (2016).

[32] S. Osher AND R. FEDKIW, Level set methods and dynamic implicit surfaces, vol. 153, Springer Science \& Business Media, 2006.

[33] N. Paragios, Y. Chen, and O. Faugeras, Handbook of mathematical models in computer vision. secaucus, 2005.

[34] F. PARK, Image segmentation using clique based shape prior and the mumford shah functional, in Image Processing (ICIP), 2015 IEEE International Conference on, IEEE, 2015, pp. 4077-4081.

[35] T. Riklin-Raviv, N. Kiryati, and N. Sochen, Prior-based segmentation and shape registration in the presence of perspective distortion, International Journal of Computer Vision, 72 (2007), pp. 309-328.

[36] M. Rousson and N. Paragios, Prior knowledge, level set representations 83 visual grouping, International Journal of Computer Vision, 76 (2008), pp. 231-243.

[37] L. I. Rudin, S. Osher, And E. FAtemi, Nonlinear total variation based noise removal algorithms, Physica D: nonlinear phenomena, 60 (1992), pp. 259-268.

[38] G. Sundaramoorthi and A. Yezzi, Global regularizing flows with topology preservation for active contours and polygons, Image Processing, IEEE Transactions on, 16 (2007), pp. 803-812.

[39] S. R. Thiruvenkadam, T. F. Chan, and B.-W. Hong, Segmentation under occlusions using selective shape prior, in International Conference on Scale Space and Variational Methods in Computer Vision, Springer, 2007, pp. 191-202.

[40] G. Vogiatzis, C. H. Esteban, P. H. Torr, and R. Cipolla, Multiview stereo via volumetric graph-cuts and occlusion robust photo-consistency, IEEE Transactions on Pattern Analysis and Machine Intelligence, 29 (2007), pp. 2241-2246. 
[41] K. Wei, K. Yin, X.-C. TAI, And T. F. Chan, New region force for variational models in image segmentation and high dimensional data clustering, arXiv preprint arXiv:1704.08218, (2017).

[42] A. J. Yezzi, S. Kichenassamy, A. Kumar, P. J. Olver, and A. R. TannenBAUM, A geometric snake model for segmentation of medical imagery, IEEE Transactions on Medical Imaging, 16 (1997), pp. 199-209.

[43] W. Zhu and T. Chan, A variational model for capturing illusory contours using curvature, J. Math. Imaging Vis., 27 (2007), pp. 29-40. 